\title{
Climate Change Solution
}

\section{William John Montague*}

Independent Researcher, Montreal, Canada

*Corresponding Author: William John Montague, Independent Researcher, Montreal, Canada.

DOI: $10.31080 /$ ASMI.2020.03.0514
Received: January 06, 2020

Published: February 08, 2020

(C) All rights are reserved by William John Montague.
What the world needs is an easily manufactured clean burning alternative to fossil fuels.

A clean burning replacement fuel can be manufactured anywhere it is needed from atmospheric nitrogen. The wealthiest and most powerful fossil fuel exporting nations do not want this knowledge to surface.

The theory, design and construction details all exist for building such an apparatus which uses rapidly changing electrostatic forces to facilitate the required dissociation of atmospheric nitrogen molecules. I am aware that modern science says there is no such thing as an alternating electrostatic force.

The underlying concepts that led to this particular technological discovery are well described in a series of lectures that Nikola Tesla (1856-1943) presented to various international scientific communities between 1891 and 1893 regarding the phenomena he investigated. Such an apparatus is also capable of dissociating carbon dioxide molecules meaning that fossil fuel combustion can continue without the emissions.

This electrical technology requires the use of very high potential differences alternating at very high frequencies to create powerful alternating electrostatic force fields between two oppositely charged conducting surfaces.

Purified atmospheric gases such as nitrogen when passed slowly through such an electrified space will accumulate enough energy for molecular dissociation to occur. This process is inexpensive because only a small amount of energy is required to create and maintain the alternating electrostatic force fields. Most of the necessary energy needed for molecular dissociation is obtained by causing molecular collisions to occur within the volume of gas.

A machine demonstrating this method of molecular gas dissociation was patented by Tesla in 1896. It was called; "Apparatus For Producing Ozone". The details needed for understanding the theory and construction are explained in a series of lectures on high voltage alternating currents that Tesla presented to American and European scientists between 1891 and 1893.

This ability to harness the power of changing electrostatic force fields came as direct result of Tesla's unique ether theory and how powerful electrical interactions affect gaseous molecular matter.

The dismal results of COP-25 show that climate change mismanagement by world powers based on preserving the power of fossil fuel exporting nations has gotten out of hand. To repeat, what the world requires now is the immediate end to fossil fuel combustion emissions. Nothing else will help. Nikola Tesla's discovery of this electrostatic technology provides a means for doing so. It only makes sense for the civilized nations of the world to use dissociated atmospheric nitrogen to provide for their own energy supply. It only makes sense to use a clean burning fuel capable of being manufactured anywhere in the world it is needed. It is well known by modern science that the only difficulty in accessing the explosive power released by combining nitrogen atoms is the energy required for the initial separation of inert diatomic nitrogen molecules.

Tesla's method and apparatus accomplishes molecular dissociation using a fraction of the energy that chemistry and physics say is necessary simply because Tesla's apparatus uses the internal en- 
ergy of the gas molecules to excite each other. His rapidly changing electrostatic force fields cause gas molecules to collide. The collisions cause internal vibrations to increase within the gas molecules to such an extent that most of the required energy needed for molecular dissociation accumulates directly at molecular boundaries. Tesla's apparatus has no need for the high temperatures and external pressure containment such as are currently required by industrial processes to make ammonia (NH3).

It was the purchase of Nikola Tesla's (1856 - 1943) alternating current motor patents in 1888 that enabled the George Westinghouse (1846 - 1914) alternating current transmission system to win the 'current wars' for providing Americans with electric lighting in the mid 1880's. The addition of a practical industrial sized motor ended the only superiority that direct current systems had left. This sale made Tesla independently wealthy and permitted him to build his laboratory and pursue his investigations and experiments into the physical nature of electricity.

Three years later in May of 1891, he first presented his findings to the American Institute of Electrical Engineers in the United Sates. Subsequent to this lecture, Tesla was invited to go over to London. In 1892 he made that journey and presented a similar lecture regarding his "Experiments with Alternate Currents of High Potential and High Frequency". The audience was composed of the leading English Electrical Engineers and Scientists of the time. He repeated this lecture a month later to a similar audience in Paris. Tesla's presented another lecture on these matters the following year in the United States.

In a continuing effort to find those persons in positions of authority who care to make this change, which I believe is the only way to save most of the life on our planet from extinction, I offer these comments on a few excerpts from these lectures.

Tesla began his London lecture with a tribute to the works of Professor William Crookes (1832 - 1919), describing the inspiration he obtained from the investigative work of Crookes into that 'radiant matter' that Micheal Faraday (1791 - 1867) was the first to call attention to back around 1820. Faraday described a fourth state of matter which he deemed 'radiant' and as different in it's properties from a gas as a gas is to a liquid.

We now say that 'radiant matter' or what we now call 'plasma', was discovered the chemist and electrical researcher Irving Langmuir
(1881 - 1957). We now say Plasma is a charged gas, with strong Coulomb [or electrostatic] interactions. Atoms or molecules acquire a positive or negative electrical charge when they gain or lose electrons. This process is called ionization. Plasma makes up the sun and stars, and it is the most common state of matter in the universe as a whole.

Tesla's unprecedented results come from conducting his experimentation on as large a scale as he could which meant at standard atmospheric conditions. The effects on atmospheric gases are the pertinent remarkable results of his experiments.

Professor Crookes, in his lecture on radiant matter presented to this same scientific body in 1879 and almost six decades after Faraday's first observations, ended his discourse on Radiant Matter with the following quote:

"Yet all these were, when no man did them know,

Yet have from the wisest ages hidden beene,

And later times thinges more unknowne shall show.

Why then should witlesse man so much misweene,

That nothing is, but that which he has seene!"

An excerpt from "The Faerie Queene", by Edmund Spenser (The Quest of Sir Guyon).

In the last few pages of his paper, Professor Crookes explains his investigations and conclusions regarding the Chemistry of Radiant Matter some of which I reference further on. What we now know as the 'electron', was only officially 'discovered' in 1897 by J.J. Thompson (1856 - 1940) almost twenty years after Crookes's cathode ray particle experiments and another five years after Tesla's lectures describing his high voltage experiments.

What I am saying is that Tesla's machine is simply far more efficient than anything that anyone else has ever built to accomplish this specific work. Simply because no one understands his theories and how to use electricity safely to generate the necessarily powerful electrostatic forces required does not make it wrong. Tesla accomplished this work through his unique understanding of how electricity and matter and the fundamental underlying ether of 'not so empty space', interact.

This being said, I would draw to your attention that Tesla created a powerful and rapidly changing electrostatic force field as a

Citation: William John Montague. "Climate Change Solution". Acta Scientific Microbiology 3.3 (2020): 01-09. 
background environment. He did this by creating very high voltages and then alternating them at extremely high frequencies. It is only because he creates this powerful background electric field environment that his apparatus so efficiently dissociates linear atmospheric gas molecules at 'normal temperatures and pressures' and without the expenditure of a lot of energy.

According to modern physics, there is no such thing as an alternating electrostatic field. The mathematics and formulas of our modern electrical engineering are well proven to confirm this. The possibilities of such alternating electrostatic fields as Tesla describes are simply are not accounted for nor recognized by our 'current', electrical laws of chemistry and physics. In our modern world of scientific consensus, the governing bodies unanimously agree that our mathematically proven electrical laws of science are correct. I suggest that our modern electrical laws were all 'tailored' to make electricity safer and for easier economic controls by conducting electricity through wires and transmission lines.

All of our modern electrical laws are based on Ampere's current law which breaks down in such an environment as Tesla creates. This means the formulas no longer produce meaningful or accurate results in Tesla's apparatus. Failure to understand Tesla's unique perspective on electrical science leads directly to catastrophic results. What I mean is that every time anyone has tried to replicate Tesla's work using modern theoretical concepts, the machines either blow up or melt down. Unless Tesla's instructions are adhered to it will not be possible to replicate his work.

The crux of the matter is simply my claim that Tesla's method and apparatus is capable of effecting the dissociation of linear atmospheric gas molecules at what we consider normal atmospheric temperatures and pressures using much less energy to accomplish this than than modern physics says is possible. I am not proclaiming an over unity generator. I am not proclaiming a perpetual motion machine. I am not saying that Tesla's machine will perform anything that modern science is not very well aware of.

In the introduction to his first U.S. Lecture in 1891, Tesla speculated on what exactly electricity is:

Of all the forms of nature's immeasurable, all-pervading energy, which ever and ever changing and moving; like a soul animates the inert universe, electricity and magnetism are perhaps the most fascinating. The effects of gravitation, of heat and light we observe daily, and soon we get accustomed to them, and soon they lose for us the character of the marvelous and wonderful; but electricity and magnetism, with their singular relationship, with their seemingly dual character, unique among the forces in nature, with their phenomena of attractions, repulsions and rotations, strange manifestations of mysterious agents; stimulate and excite the mind to thought and research. What is electricity, and what is magnetism? These questions have been asked again and again. The most able intellects have ceaselessly wrestled with the problem; still the question has not as yet been fully answered. But while we cannot even to-day state what these singular forces are, we have made good headway towards the solution of the problem. We are now confident that electric and magnetic phenomena are attributable to ether, and we are perhaps justified in saying that the effects of static electricity are effects of ether under strain, and those of dynamic electricity and electro-magnetism effects of ether in motion. But this still leaves the question, as to what electricity and magnetism are, unanswered.

First, we naturally inquire, What is electricity, and is there such a thing as electricity? In interpreting electric phenomena: we may speak of electricity or of an electric condition, state or effect. If we speak of electric effects we must distinguish two such effects, opposite in character and neutralizing each other, as observation shows that two such opposite effects exist. This is unavoidable, for in a medium of the properties of ether, we cannot possibly exert a strain, or produce a displacement or motion of any kind, without causing in the surrounding medium an equivalent and opposite effect. But if we speak of electricity, meaning a thing, we must, I think, abandon the idea of two electricities, as the existence of two such things is highly improbable. For how can we imagine that there should be two things, equivalent in amount, alike in their properties, but of opposite character, both clinging to matter, both attracting and completely neutralizing each other? Such an assumption, though suggested by many phenomena, though most convenient for explaining them, has little to commend it. If there is such a thing as electricity, there can be only one such thing, and; excess and want of that one thing, possibly; but more probably its condition determines the positive and negative character. The old theory of Franklin, though falling short in some respects; is, from a certain point of view, after all, the most plausible one. Still, in spite of this, the theory of the two electricities is generally accepted, as it apparently explains electric phenomena in a more

Citation: William John Montague. "Climate Change Solution". Acta Scientific Microbiology 3.3 (2020): 01-09. 
satisfactory manner. But a theory which better explains the facts is not necessarily true. Ingenious minds will invent theories to suit observation, and almost every independent thinker has his own views on the subject.

It is not with the, object of advancing an opinion; but with the desire of acquainting you better with some of the results, which I will describe, to show you the reasoning I have followed, the departures I have made-that I venture to express, in a few words, the views and convictions which have led me to these results.

I adhere to the idea that there is a thing which we have been in the habit of calling electricity. The question is, What is that thing? or, What, of all things, the existence of which we know, have we the best reason to call electricity? We know that it acts like an incompressible fluid; that there must be a constant quantity of it in nature; that it can be neither produced nor destroyed; and, what is more important, the electro-magnetic theory of light and all facts observed teach us that electric and ether phenomena are identical. The idea at once suggests itself, therefore, that electricity might be called ether. In fact, this view has in a certain sense been advanced by Dr. Lodge. His interesting work has been read by everyone and many have been convinced by his arguments. Isis great ability and the interesting nature of the subject, keep the reader spellbound; but when the impressions fade, one realizes that he has to deal only with ingenious explanations. I must confess, that I cannot believe in two electricities, much less in a doubly-constituted ether. The puzzling behavior of tile ether as a solid waves of light anti heat, and as a fluid to the motion of bodies through it, is certainly explained in the most natural and satisfactory manner by assuming it to be in motion, as Sir William Thomson has suggested; but regardless of this, there is nothing which would enable us to conclude with certainty that, while a fluid is not capable of transmitting transverse vibrations of a few hundred or thousand per second, it might not be capable of transmitting such vibrations when they range into hundreds of million millions per second. Nor can anyone prove that there are transverse ether waves emitted from an alternate current machine, giving a small number of alternations per second; to such slow disturbances, the ether, if at rest, may behave as a true fluid.

Returning to the subject, and bearing in mind that the existence of two electricities is, to say the least, highly improbable, we must remember, that we have no evidence of electricity, nor can we hope to get it, unless gross matter is present. Electricity, therefore, cannot be called ether in the broad sense of the term; but nothing would seem to stand in the way of calling electricity ether associated with matter, or bound ether; or, in other words, that the so-called static charge of the molecule is ether associated in some way with the molecule. Looking at it in that light, we would be justified in saying, that electricity is concerned in all molecular actions.

Now, precisely what the ether surrounding the molecules is, wherein it differs from ether in general, can only be conjectured. It cannot differ in density, ether being incompressible; it must, therefore, be under some strain or is motion, and the latter is the most probable. To understand its functions, it would be necessary to have an exact idea of the physical construction of matter, of which, of course, we can only form a mental picture.

But of all the views on nature, the one which assumes one matter and one force, and a perfect uniformity throughout, is the most scientific and most likely to be true. An infinitesimal world, with the molecules and their atoms spinning and moving in orbits, in much the same manner as celestial bodies, carrying with them and probably spinning with them ether, or in other words; carrying with them static charges, seems to my mind the most probable view, and one which, in a plausible manner, accounts for most of the phenomena observed. The spinning of the molecules and their ether sets up the ether tensions or electrostatic strains; the equalization of ether tensions sets up ether motions or electric currents, and the orbital movements produce the effects of electro and permanent magnetism

About fifteen, years ago, Prof. Rowland demonstrated a most interesting and important fact; namely, that a static charge carried around produces the effects of an electric current. Leaving out of consideration the precise nature of the mechanism, which produces the attraction and repulsion of currents, and conceiving the electrostatically charged molecules in motion, this experimental fact gives us a fair idea of magnetism. We can conceive lines or tubes of force which physically exist, being formed of rows of directed moving molecules; we can see that these lines must be closed, that they must tend to shorten and expand, etc. It likewise explains in a reasonable way, the most puzzling phenomenon of all, permanent magnetism, and, in general, has all the beauties of the Ampere theory without possessing the vital defect of the same, namely, the assumption of molecular currents. Without enlarging further upon 
the subject, I would say, that I look upon all electrostatic, current and magnetic phenomena as being due to electrostatic molecular forces.

The preceding remarks I have deemed necessary to a full understanding; of the subject as it presents itself to my mind.

Professor Thomas H. Blakesley (1847-1929) in his book "Papers On Alternating Currents of Electricity For The Use of Students and Engineers" makes reference to the omitted mathematics for anyone interested.

To repeat, in the quotation above from Tesla's 1891 lecture to the American Society of Electrical Engineers which inspired the British invitation to cross the Atlantic and come to London, he makes reference to the importance of the experiments of American Physicist Henry Augustus Rowland (1848-1901) "a static charge carried around produces the effects of an electric current ... has all the beauties of the Ampere theory without possessing the vital defect of the same, namely, the assumption of molecular currents. Without enlarging further upon the subject, I would say, that I look upon all electrostatic, current and magnetic phenomena as being due to electrostatic molecular forces.

Rowland was another early electrical investigator and experimental physicist professor who adhered to Maxwell's (James Clerk Maxwell 1831 - 1879) mathematics but like Tesla also questioned some of his theories. They both formed their own opinions from devising their own experiments based on Faraday's observations. Faraday's thoughts and experiments seem traceable back to Boscovich's (Roger Joseph Boscovich 1711 - 1787) extensions to Newton's (Issac Newton 1643 - 1727) original ether conceptions.

To understand Tesla's work and how it is applicable to delivering the world from the fate of fossil fuel carbon dioxide emissions destruction, we need to look a whole lot deeper into electricity than just how we conduct it through wires. None of us can see any of these things with our eyes because they are all so small and so we need a proper conceptual understanding to enable us to interact more directly with them. Even now in 2020 the best magnification we can accomplish simply makes atomic matter look like fuzzy little balls. We must put aside our modern notions and go back to Maxwell's 'original' equations for electrostatic phenomena and follow Tesla's sound wave 'ether' model to understand and replicate the necessary apparatus if we want to effect the necessary molecu- lar dissociation.

To quote from Professor Crookes in his lecture 1879 lecture on radiant matter

The chemistry of radiant matter

As might be expected, the chemical distinctions between one kind of Radiant Matter and another at these high exhaustions are difficult to recognise. The physical properties I have been elucidating seem to be common to all matter at this low density. Whether the gas originally under experiment be hydrogen, carbonic acid, or atmospheric air, the phenomena of phosphorescence, shadows, magnetic deflection, and c.,are identical, only they commence at different pressures. Other facts however, show that at this low density the molecules retain their chemical characteristics. Thus by introducing into the tubes appropriate absorbents of residual gas, I can see that chemical attraction goes on long after the attenuation has reached the best stage for showing the phenomena now under illustration, and I am able by this means to carry the exhaustion to much higher degrees than I can get by mere pumping. Working with aqueous vapour I can use phosphoric anhydride as an absorbent; with carbonic acid, potash; with hydrogen, palladium; and with oxygen, carbon, and then potash. The highest vacuum I have yet succeeded in obtaining has been the $1-20,000,000^{\text {th }}$ of an atmosphere, a degree which may be better understood if I say that it corresponds to about the hundredth of an inch in a barometric column three miles high.

It may be objected that it is hardly consistent to attach primary importance to the presence of Matter, when I have taken extraordinary pains to remove as much Matter as possible from these bulbs and these tubes, and have succeeded so far as to leave only about the one-millionth of an atmosphere in them. At its ordinary pressure the atmosphere is not very dense, and its recognition as a constituent of the world of Matter is quite a modern notion (note that this was written more than 140 years ago). It would seem that when divided by a million, so little Matter will necessarily be left that we may justifiably neglect the trifling residue and apply the term vacuum to space from which the air has been so nearly removed. To do so, however, would be a great error, attributable to our limited faculties being unable to grasp high numbers. It is generally taken for granted that when a number is divided by a million the quotient must necessarily be small, whereas it may happen that the original number is so large that its division by a million 
seems to make little impression on it. According to the best authorities, a bulb of the size of the one before you contains more than 1,000000,000000,000000,000000 (a quadrillion) molecules. Now, when exhausted to a millionth of an atmosphere we shall still have a trillion molecules left in the bulb a number quite sufficient to justify me in speaking of the residue as matter.

Please also note that back in these times, the term million meant 6 zeros, billion meant 12 zeros, trillion meant 18 and quadrillion meant 24 . In our modern physics, a quadrillion has been redefined and reduced to having only 15 zeros.

Crookes goes on to say that: at the rate of $100,000,000$ or one hundred million molecules per second it would take longer than the length of time of all existence of the earth and our solar system to fill up this tiny sphere if it were empty and that because in reality it only takes a few seconds to fill the empty spherical bulb or tube through even the smallest possible tiny pin hole he could make, the molecules had to be entering at a rate of about $300,000000,000000,000000$ or three hundred trillion per second. These figures when they mount so high cease to have any meaning, and such calculations are as futile as trying to count the drops in the ocean.

\section{He concludes with}

"In studying this fourth state of matter we seem, at length, to have within our grasp and obedient to our control the little indivisible particles which, with good warrant, are supposed to constitute the physical basis of the universe. We have seen that, in some of its properties, radiant matter is as solid as this table, while in other properties it almost assumes the character of radiant energy. We have actually touched the border-land where matter and force seem to merge into one another, the shadowy realm between known and unknown, which for me has always held peculiar temptations. I venture to think that the greatest scientific problems of the future will find their solution in this border-land, and even beyond ; here, it seems to me, lie ultimate realities, subtile, far-reaching, wonderful."

Tesla always made use of cgs electrostatic units in his high voltage and high frequency research. He was only able to conduct this research because he was able to separate the electrostatic electric field effects from the electromagnetic effects or what we call electrodynamics. Applicable electrical formula's as we understand them in modern International System of units of measure will not suffice.

Tesla found that he needed very thin wires because of the effects of self induction on thicker wires by extremely high voltages and rates of change. By using very high voltages and frequencies he was able to use very small and inexpensive condensers to transmit the incredible amounts of power that only condensers are capable of, even when compared to heavy cables of inappreciable resistance.

He created unprecedented rates of change for accumulations of electrical charges by which he was able to interact more directly with molecular matter.

Tesla left behind the inductive magnetic forces governing the electromagnetic interactions of electric currents and conducting matter by making an environment where the secondary forces of electromagnetic currents were incapable of reacting because of the excessive rates of change and inductive effects of his powerful electric fields. This separation of electric and magnetic reactions are why Tesla considered electrostatic electrical forces to be the fundamental force in the physics of what we call electricity.

Tesla's apparatus more directly interacts with molecular boundaries using dielectric polarization and molecular collisions to raise the vibration temperatures directly at molecular boundaries. The collisions are how his machine effects dissociation of pure atmospheric gases so easily. He tuned his circuits like a musical instrument, adjusting the vibrations to the correspond to the point where energy transfer excites resonance leading to molecular dissociation. These concepts are well known to modern science what is different is the scale at which Tesla apparatus is able to safely control the process.

Further into the introduction to his London lecture, Tesla continues: "We observe how the energy of an alternating current traversing the wire manifests itself - not so much in the wire as in the surrounding space - in the most surprising manner, taking the forms of heat, light, mechanical energy, and, most surprisingly of all, chemical affinity.

Much of this lecture and many other of Tesla's subsequently published documents require looking up old definitions to words because they had different meanings at that time. In the electrical sciences, the definitions and meanings and agreed upon terminol- 
ogy and formulas have changed more than once since Tesla's documents were authored. Our modern understanding of electricity is much different from that of more than a hundred and fifty years ago when Crookes and Tesla and this audience of their contemporaries were all educated in possibilities. Words such as molecule and atom and chemical affinity and plasma and electron are important noteworthy examples, as are the concepts of ether and the debates about two versus one distinct forms of electricity or positive and negative electric fluids. All of this leads us to Tesla's ether concepts for the structure of empty space. If space is truly empty, then what are the things we call the Permittivity and Permeability of the vacuum of space. If the best conclusion of modern physics is that ninety six percent of the mass of the universe is missing, this can only mean that our concepts of astrophysics are hopelessly wrong. It seems to me that Crookes's explanation of gas molecules and atmospheric pressure as mentioned above may also account for much of that missing matter and that space is not as 'empty' as we are taught to conceive. Tesla said we only have the possibility of the manifestation of any electrical effects in the presence of atomic or molecular substances or what he called 'gross' matter.

In his second American lecture; "On Light And Other High Frequency Phenomena" presented in 1893, Tesla describes the different methods and the difficulties he encountered in creating the high voltages and frequencies or rates of change required for generating the different effects. Molecular dissociation is only one of many discoveries that Tesla made from these investigations. Having described much detail regarding the generation of the different electrical activities, he goes into the specific effects. Of interest to us regarding molecular dissociation are some of what he calls the electrostatic phenomena:

The first class of effects I intend to show you are effects produced by electrostatic force. It is the force which governs the motion of the atoms, which causes them to collide and develop the life-sustaining energy of heat and light, and which causes them to aggregate in an infinite variety of ways, according to Nature's fanciful designs, and to form all these wondrous structures we perceive around us; it is, in fact, if our present views be true, the most important force for us to consider in. Nature. As the term electrostatic might imply a steady electric condition, it should be remarked, that in these experiments the force is not constant, but varies at a rate which may be considered moderate, about one million times a second, or thereabouts. This enables me to produce many effects which are not producible with an unvarying force.

When two conducting bodies are insulated and electrified, we say that an electrostatic force is acting between them. This force manifests itself in attractions, repulsions and stresses in the bodies and space or medium without. So great may be the strain exerted in the air, or whatever separates the two conducting bodies, that it may break down, and we observe sparks or bundles of light or streamers, as they are called. These streamers form abundantly when the force through the air is rapidly varying.

In his summation at the end of this presentation, tesla continues

Coming now to the most interesting of these phenomena, the incandescence or phosphorescence of gases, at low pressures or at the ordinary pressure of the atmosphere, we must seek the explanation of these phenomena in the same primary causes, that is, in shocks or impacts of the atoms. Just as molecules or atoms beating upon a solid body excite phosphorescence in the same or render it incandescent, so when colliding among themselves they produce similar phenomena. But this is a very insufficient explanation and concerns only the crude mechanism. Light is produced by vibrations which go on at a rate almost inconceivable. If we compute, from the energy contained in the form of known radiations in a definite space the force which is necessary to set up such rapid vibrations, we find, that though the density of the ether be incomparably smaller than that of any body we know, even hydrogen, the force is something surpassing comprehension. What is this force, which in mechanical measure may amount to thousands of tons per square inch? It is electrostatic force in the light of modern views. It is impossible to conceive how a body of measurable dimensions could be charged to so high a potential that the force would be sufficient to produce these vibrations. Long before any such charge could be imparted to the body it would be shattered into atoms. The sun emits light and heat, and so does an ordinary flame or incandescent filament, but in neither of these can the force be accounted for if it be assumed that it is associated with the body as a whole. Only in one way may we account for it, namely, by identifying it with the atom. An atom is so small, that if it be charged by coming in contact with an electrified body and the charge be assumed to follow the same law as in the case of bodies of measurable dimensions, it must retain a quantity of electricity which is fully capable of accounting for these forces and tremendous rates of vibration. But the atom behaves singularly in 
this respect-it always takes the same "charge".

It is very likely that resonant vibration plays a most important part in all manifestations of energy in nature. Throughout space all matter is vibrating, and all rates of vibration are represented, from the lowest musical note to the highest pitch of the chemical rays, hence an atom, or complex of atoms, no matter what its period, must find a vibration with which it is in resonance. When we consider the enormous rapidity of the light vibrations, we realize the impossibility of producing such vibrations directly with any apparatus of measurable dimensions, and we are driven to the only possible means of attaining the object of setting up waves of light by electrical means and economically, that is, to affect the molecules or atoms of a gas, to cause them to collide and vibrate. We then must ask ourselves-How can free molecules or atoms be affected? It is a fact that they can be affected by electrostatic force, as is apparent in many of these experiments. By varying the electrostatic force we can agitate the atoms, and cause them to collide accompanied by evolution of heat and light. It is not demonstrated beyond doubt that vie can affect them otherwise. If a luminous discharge is produced in a closed exhausted tube, do the atoms arrange themselves in obedience to any other but to electrostatic force acting in straight lines from atom to atom?.

Furthermore, with my present experience I do not think that such a gas discharge in a closed tube can vibrate, that is, vibrate as a whole. I am convinced that no discharge through a gas can vibrate. The atoms of a gas behave very curiously in respect to sudden electric impulses. The gas does not seem to possess any appreciable inertia to such impulses, for it is a fact, that the higher the frequency of the impulses, with the greater freedom does the discharge pass through the gas. If the gas possesses no inertia then it cannot vibrate, for some inertia is necessary for the free vibration. I conclude from this that if a lightning discharge occurs between two clouds, there can be no oscillation, such as would be expected, considering the capacity of the clouds. But if the lightning discharge strike the earth, there is always vibration-in the earth, but not in the cloud. In a gas discharge each atom vibrates at its oven rate, but there is no vibration of the conducting gaseous mass as a whole. This is an important consideration in the great problem of producing light economically, for it teaches us that to reach this result we must use impulses of very high frequency and necessarily also of high potential.
Much would remain to be said about the luminous effects produced in gases at low or ordinary pressures. With the present experiences before us we cannot say that the essential nature of these charming phenomena is sufficiently known.

\section{Tesla concludes this lecture as follows}

In presenting these insignificant results I have not attempted to arrange and coordinate them, as would be proper in a strictly scientific investigation, in which every succeeding result should be a logical sequence of the preceding, so that it might be guessed in advance by the careful reader or attentive listener. I have preferred to concentrate my energies chiefly upon advancing novel facts or ideas which might serve as suggestions to others, and this may serve as an excuse for the lack of harmony. The explanations of the phenomena have been given in good faith and in the spirit of a student prepared to find that they admit of a better interpretation. There can be no great harm in a student taking an erroneous view, but when great minds err, the world must dearly pay for their mistakes.

As to the devices, Tesla's authored many articles, books, patents and lectures all of which provide descriptions for all the necessary connections and parts. His rotary turbine which he referred to as the 'monarch of machines' also has electrical purposes as does his valvular conduit design. Tesla intentionally left cryptic instructions for building electrostatic generators of unprecedented power. His final antenna design patent shows the methods of safely connecting high power circuits. Tesla left very specific instructions on how and when to use self induction instead of resistance so as to avoid energy losses or how to use distributed capacity to overcome self induction and transfer energy by pumping it up and letting it fall. He uses symmetry and reciprocation and explains the requirements of how to calculate the construction of appropriate inductors and condensers needed to accumulate and transfer the necessary electrical charges. He uses resonance and interference and reflection throughout his designs. All the required notes and formulas are well described in his body of published works including numerous specific reference instructions for effecting the dissociation of linear molecular gases.

Once again, I offer my assistance to anyone, anywhere who wants to prevent the devastation $\mathrm{n}$ that the continuation of following the energy policies designed to preserve the supremacy and wealth and monopolies of fossil fuel economics.

Citation: William John Montague. "Climate Change Solution". Acta Scientific Microbiology 3.3 (2020): 01-09. 
The entire world is rapidly running out of time.

\section{Assets from publication with us}

- Prompt Acknowledgement after receiving the article

- Thorough Double blinded peer review

- Rapid Publication

- Issue of Publication Certificate

- High visibility of your Published work

Website: https://www.actascientific.com/

Submit Article: https://www.actascientific.com/submission.php

Email us: editor@actascientific.com

Contact us: +919182824667 